\title{
QUARANTE ANNÉES D'IMAGERIE SATELLITAIRE RADAR
}

\author{
Jean Marie Nicolas ${ }^{1}$, Florence Tupin ${ }^{1}$ \\ 1 : LTCI, Télécom Paris, Institut Polytechnique de Paris, 75013, Paris, France
}

\begin{abstract}
Résumé
Depuis le lancement de Seasat en 1978, les satellites RSO (Radar à Synthèse d'Ouverture) fournissent des images qui sont devenues au fil des années de plus en plus résolues et nombreuses. Cet article a pour but de montrer certaines évolutions, tant technologiques que sur le plan des traitements, qui ont jalonné ces quarante dernières années et qui ont permis d'avoir des données actuellement incontournables en télédétection.
\end{abstract}

\section{Abstract}

Since Seasat launching in 1978, SAR satellites provides data which are more and more accurate and numerous over the years. This article aims to emphasize as well some technological evolutions of SAR sensors as some improvments in SAR image processing over the last 40 years so that these data have now an essential role in remote sensing.

\section{Introduction}

De nos jours, la télédétection propose une large gamme de capteurs en orbite autour de la Terre. Parmi ceux-ci les systèmes RSO (Radar à Synthèse d'Ouverture), appelés SAR par les anglo-saxons (Synthetic Ar-ray Radar) sont devenus une modalité incontournable et d'autant plus accessible que plusieurs types de RSO permettent aux thématiciens d'accéder à des images diverses : couples interférométriques pour le suivi de la croûte terrestre, polarimétriques pour la classification des surfaces végétales, piles temporelles (stack) pour la détection de changements.

Face à cette profusion actuelle de données, il est facile d'oublier le long cheminement qui a couvert un demi-siècle et qui, d'un capteur expérimental (Seasat), nous a amenés à des systèmes à faible temps de revisite, alliant aussi bien haute résolution (métrique) mais faible couverture spatiale (TerraSAR-X, Cosmo-Skymed), que grande couverture spatiale avec une résolution de moindre performance (Sentinel-1). Or, beaucoup d'expérimentations, principalement menées à la charnière de ce millénaire, ont conduit à la situation actuelle : il était donc intéressant d'en évaluer leurs portées.

Le présent article vise d'une part à montrer les évolutions matérielles qui, grâce aux progrès toujours rapides de l'électronique embarquée, ont conduit à la mise en orbite de capteurs métriques, et d'autre part à illustrer les évolutions les plus récentes dans les traitements des données RSO, d'autant plus délicates à traiter que le bruit, lié au phénomène de chatoiement présent sur ce type d'image, y a un caractère multiplicatif et requiert des outils statistiques spécifiques.

\section{Un demi-siècle de capteurs radar}

L'imagerie RSO (Radar à Synthèse d'Ouverture), génialement proposée par Wiley en 1951, a véritablement vécu ses heures de gloire quand le JPL a mis en orbite un satellite civil d'observation de la Terre équipé d'un tel système : c'était le satellite Seasat en 1978 , soit il y a près de quarante ans.
Depuis, les progrès de l'électronique ont mené ce type de capteur à une maturité à laquelle ne songeaient peut être pas les concepteurs de Seasat : en 2016, ce n'est pas moins de douze systèmes RSO qui imagent notre planète..

En raison des caractéristiques électroniques requises pour de tels systèmes (nécessité de "spatialiser" les composants électroniques par exemple) et de la complexité pour définir et mener à bien le lancement de nouvelles missions spatiales, les progrès en presque un demi-siècle n'ont pas été continus : on peut dire qu'en pratique ont été mises à la disposition des scientifiques trois générations de satellites, chacune avec des caractéristiques spécifiques. Ce paragraphe a pour vocation de proposer une telle classification qui, même arbitraire, met en valeur les progrès accomplis.

\subsection{La première génération de capteurs : 1978-2002}

\subsubsection{Le pionnier: Seasat (1978)}

Dans le cadre des missions dédiées à la surveillance des océans, le JPL a mis en orbite le premier satellite civil dont un des équipements était un capteur radar à synthèse d'ouverture. Avec son antenne monolithique et son électronique analogique, il a été une parfaite illustration du principe d'acquisition stripmap. En effet, à partir de la dimension de l'antenne $L$ et la longueur d'onde $\lambda$, l'ouverture angulaire (appelée aussi lobe d'antenne) s'écrit :

$$
\omega=2 \frac{\lambda}{L}
$$

(le facteur 2 venant de ce que l'on se place entre les premiers zéros de l'antenne physique). Connaissant cette ouverture angulaire ainsi que la distance entre zone imagée et satellite $R$, on en déduit la relation fondamentale de la résolution $\delta y$ des systèmes cohérents :

$$
\delta y=\frac{R \omega}{2}=\frac{\lambda R}{L}
$$


qui exprime que l'amélioration de la résolution passe par une augmentation de la dimension de l'antenne. Pour Seasat, la dimension de son antenne physique le long de la direction "azimut" (10.7m, voir le tableau 1) ne lui permettait à priori qu'une résolution kilométrique. Grâce au principe de la synthèse d'ouverture (voir par exemple (Massonnet et Souyris, 2008)) qui nécessite, dans sa modalité la plus simple appelée stripmap, une succession d'acquisitions identiques le long de la trajectoire du satellite, tout se passe comme si l'on disposait d'une antenne synthétique dont la dimension se déduit directement de la relation (1) :

$$
L_{S}=R \omega=\frac{2 \lambda R}{L}
$$

puisque tous les tirs radar pour lesquels la zone imagée est à l'intérieur du lobe d'antenne interviennent dans la synthèse au point considéré. En prenant cette valeur $L_{S}$ comme dimension d'antenne dans la relation (3), on déduit la célèbre formule de la résolution des RSO stripmap :

$$
\delta y=\frac{L}{2}
$$

Pour Seasat, on a ainsi une résolution azimutale de l'ordre de $5.3 \mathrm{~m}$, bien meilleure que celle des capteurs optiques de l'époque, c'est à dire les Landsat 1 à 3.

Pour l'axe "distance", selon lequel le radar fonctionne comme tout système d'écholocation (sonar, radar, lidar), la résolution $\delta r$ ne dépend que de la bande passante $B W$ du signal émis (voir par exemple (Massonnet et Souyris, 2008)) et elle s'écrit :

$$
\delta_{r}=\frac{c}{2 B W}
$$

ce qui donne par projection la résolution sol $\delta x$ :

$$
\delta_{x}=\frac{c}{2 B W \sin \theta}
$$

Selon cet axe distance, la fauchée, c'est à dire le lieu des points sur la Terre imageable par le radar, est déterminée par la dimension du lobe d'antenne (selon le petit côté de l'antenne: $l$ ).

Optimisé pour l'observation de la surface de la mer (ce qui impose le choix d'une polarimétrie $\mathrm{HH}-$ le radar émet en polarisation horizontale et reçoit en polarisation horizontale-, et d'incidence moyenne $-23.5^{\circ}$ au centre de l'acquisition-), Seasat a néanmoins surpris la communauté scientifique en fournissant des images de la surface continentale tout à fait exploitables par les thématiciens, en particulier grâce à ses capacités à acquérir des données indépendamment de la nébulosité (capteur "tout temps"). II est intéressant de remarquer que la dimension pixellique des produits Seasat, $12.5 \mathrm{~m} \times 12.5 \mathrm{~m}$ pour les images mises à la disposition de la communauté scientifique par l'Alaska Satellite Facility (avec un traitement multivues $L=4$ pour adoucir les effets du chatoiement) était tout à fait compétitive vis à vis des capteurs optiques en orbite à cette époque, c'est à dire le satellite Landsat-3 qui avait une résolution nominale de $80 \mathrm{~m}$ (capteur MSS). La figure 1 permet cette comparaison sur la ville de Hoorn au bord de l'ljlsmeer (Pays-Bas). Sa fauchée (de l'ordre de $110 \mathrm{~km}$ ) était aussi du même ordre de grandeur que la dimension des images Landsat-3 (180 $\mathrm{km})$.

L'expérience a malheureusement été de courte durée (108 jours) suite à un court-circuit qui mit fin aux diverses missions de ce satellite.
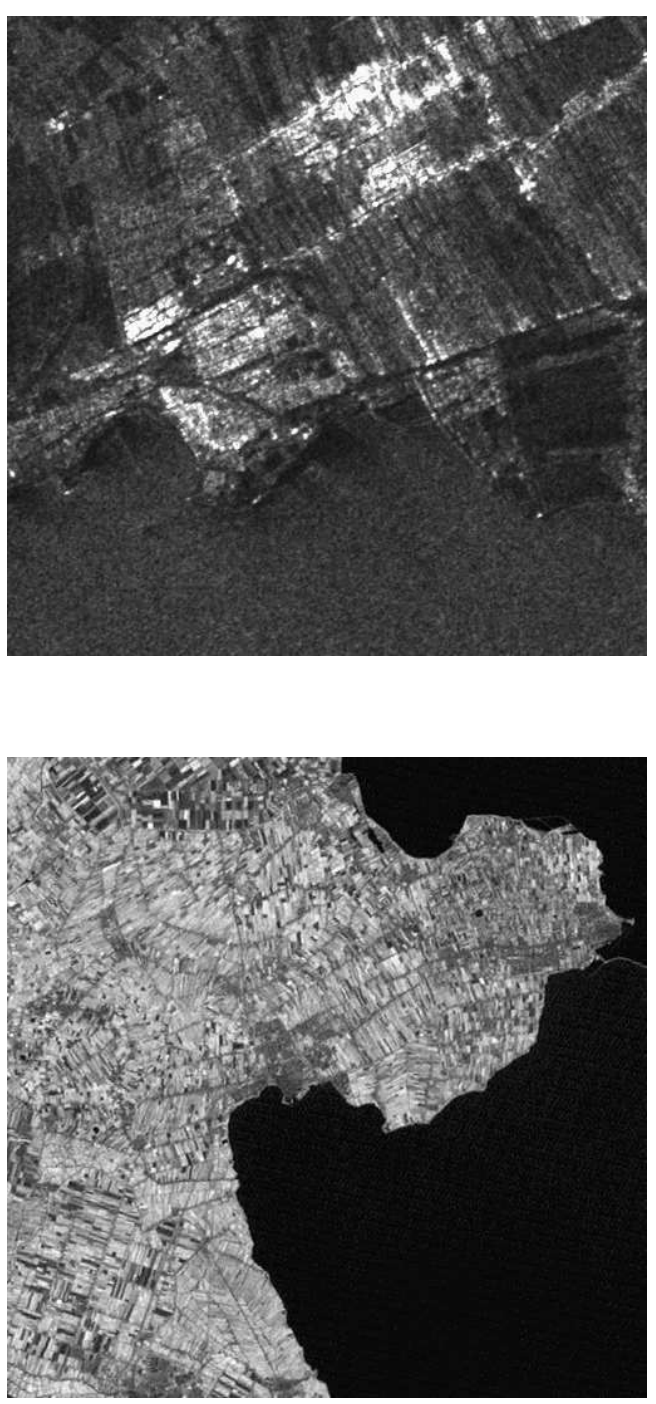

Figure 1: Image Seasat (données Seasat NASA-1978, traitées par ASF DAAC, 2013, pixel carré de $12.5 \mathrm{~m}$ ) sur la ville de Hoorn au bord de l'ljlsmeer (Pays-Bas). En bas, la même zone imagée par le capteur LANDSAT-5 en 1984 (capteur MSS, U.S. Geological Survey, pixel de $80 \mathrm{~m}$ ) : la ville de Hoorn est au centre. Les deux imagettes ont $512 \times 512$ pixels.

\subsubsection{L'ESA et les ERS (1991)}

C'est l'Agence Spatiale Européenne (ESA) qui, une bonne décennie plus tard (en 1991), a placé sur orbite le premier satellite RSO dédié à la surface continentale (bien que le choix de la polarisation VV ait été dicté pour des données acquises sur la mer) et qui a ainsi distribué à la communauté scientifique des données RSO dont la résolution était finalement assez proche des données Seasat. En une bonne dizaine d'années, les progrès dans 
les systèmes numériques ont permis d'avoir une numérisation à bord, même si les convertisseurs analogiquenumérique disponibles à cette époque et les capacités de transfert numérique sur Terre avait eux aussi des limitations intrinsèques à la technologie des années 90 . C'est pour cela que finalement les images ERS ont une résolution largement décamétrique tout à fait comparable au système Seasat, bien moins bonne que la résolution quasi décamétrique des données SPOT (le premier, SPOT-1, ayant été lancé en 1986). La figure 2 compare une imagette ERS avec une imagette Landsat-7 prises sur la ville de Hoorn au bord de l'ljlsmeer (Pays-Bas).
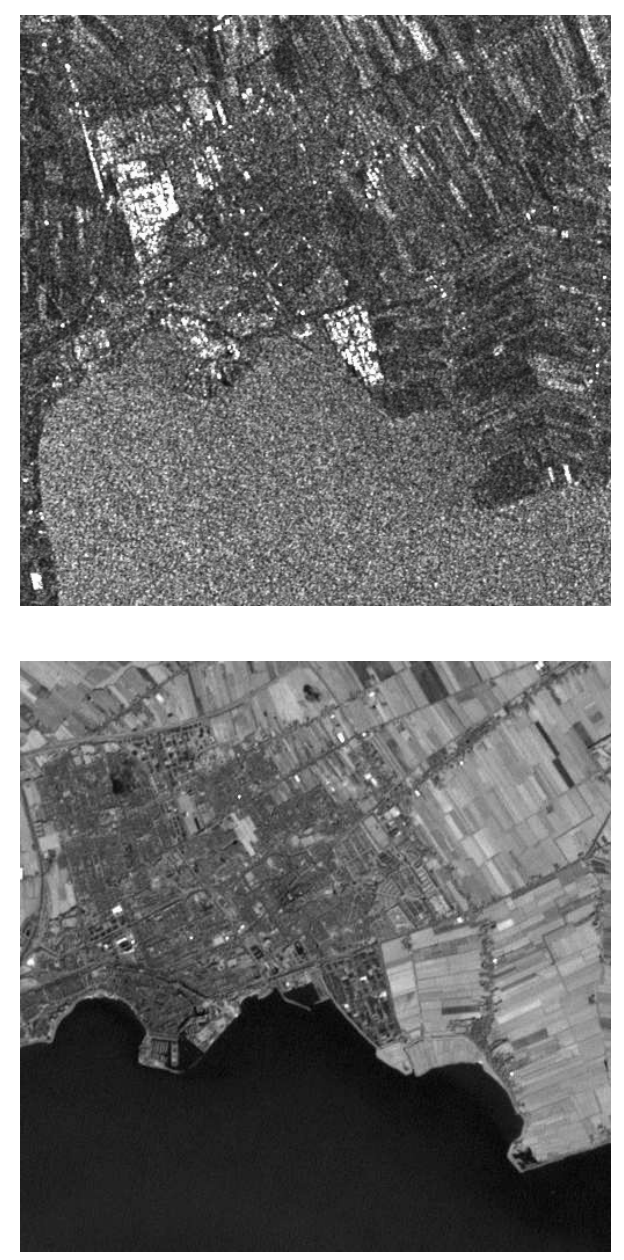

Figure 2 : Image ERS sur la ville de Hoorn au bord de l'llismeer (Pays-Bas) : la dimension du pixel de cette imagette est environ $20 \mathrm{~m}$. En bas, la même zone imagée par le capteur LANDSAT-7 (pixel de $15 \mathrm{~m}$ ). Les deux imagettes ont $512 \times 512$ pixels.

Comme clans le cas ce sasat, l'antenne est monolithique, c'est à dire qu'à l'émission et à la réception, le signal est intégré sur la surface entière de l'antenne sans possibilité d'adjoindre un processus de focalisation : le lobe d'antenne est alors perpendiculaire à la surface de l'antenne et ne peut être modifié. La différence avec Seasat est la bande utilisée : la bande C (longueur d'onde $5.66 \mathrm{~cm}$ ). Un des éléments essentiels du capteur ERS est dans sa géométrie d'acquisition qui est fixe : l'inci- dence au centre de l'image est d'environ $23^{\circ}$, et, en absence de toute agilité du système, la répétitivité des acquisitions sur un site donné (c'est à dire le temps de revisite) correspond exactement au cycle du satellite c'est à dire 35 jours. Grâce à ces choix figés, un catalogue de données prises quasiment selon la même géométrie a été constitué au fil des ans, d'autant plus qu'un second satellite identique (ERS-2) a été mis en orbite en 1995 et que la fiabilité a été assez exemplaire pour l'époque (9 ans pour ERS-1, 16 ans pour ERS-2).

Une des conséquences les plus marquantes de ce catalogue réside dans les manières possibles d'exploiter une pile d'images sur un site donné. Si le traitement des amplitudes est une première manière d'évaluer les changements temporels, le fait que les données soient des valeurs complexes permet d'exploiter les phases et plus précisément les différences de phase : c'est le domaine de l'interférométrie (Tupin et al., 2013). Notons que ces différences n'ont un sens qu'à condition que les horloges embarquées aient une excellente précision. Ces différences de phase, qui correspondent donc à des différences de trajet de l'ordre de la longueur d'onde, sont principalement liées à la très légère différence de géométrie d'acquisition, le pilotage des satellites garantissant leurs passages autour d'une orbite de référence à une distance de l'ordre de la centaine de mètres (pour une distance à la zone imagée de l'ordre du millier de kilomètres).

Grâce au catalogue de données ERS, des analyses en configuration interférométrique extrêmement probantes ont pu être obtenues par les chercheurs et ont ainsi promu cette technique pour construire des Modèles Numériques de Terrain (MNT) et pour effectuer des mesures de subsidence de la surface terrestre.

\subsubsection{Le Japon et la bande L (1992)}

L'anné suivante (1992) c'est l'agence spatiale japonaise qui met en orbite JERS, un radar en bande $L$ avec les mêmes limitations en résolution qu'ERS et les mêmes contraintes (géométrie fixe, cycle de 44 jours). Les résultats obtenus sur les zones forestières pendant les six années de son fonctionnement ont montré tout l'intérêt de la bande $L$ pour le suivi temporel de ce type de surface.

\subsubsection{Le Canada et l'avènement des antennes} paramétrisables (1995)

En 1995, l'agence spatiale canadienne innove avec la mise en orbite d'une antenne radar à focalisation électronique : il devient possible de paramétrer l'incidence locale. Le cycle du satellite est diminué (25 jours), et l'agilité permet de diminuer le temps de revisite (ce que les satellites SPOT faisaient dès 1986).

L'idée est de remplacer l'antenne monolithique par des éléments de base pavant cette antenne selon la direction de déplacement du satellite et dont la phase peut être ajustée de sorte que l'angle d'émission de l'onde peut varier sans mouvement de la plateforme (voir figure 3). 


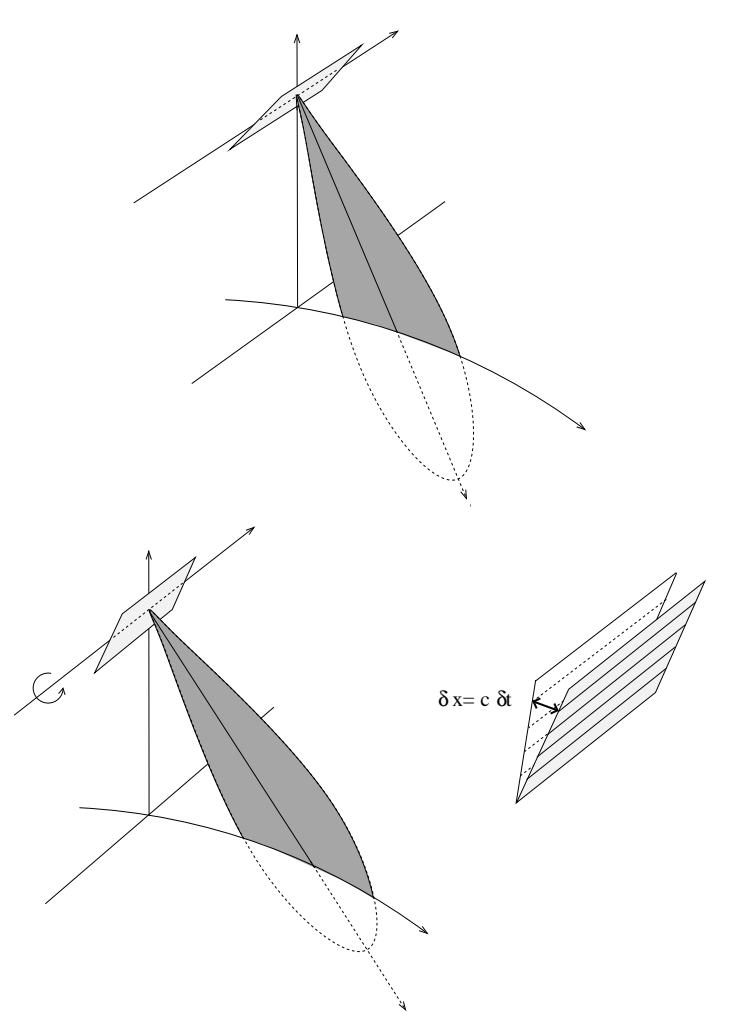

Figure 3 : En haut, antenne radar "monolithique" : à l'émission, l'état ondulatoire est identique en tout point de l'antenne et toute modification éventuelle de l'incidence locale est obtenue par basculement de la plateforme. En bas : antenne à focalisation électronique. En ajustant les retards (par simple déphasage) sur des bandes parallèles au déplacement du capteur, tout se passe comme si on basculait en élévation le faisceau émis. Ainsi on si-mule un dépointage mécanique de l'antenne et on offre une agilité d'angle d'émission selon l'élévation au système radar.

En bande C comme ERS (mais avec une polarisation différente), et grâce à cette possibilité d'acquérir des images d'une même zone selon des incidences différentes, Radarsat-1 a permis de valider le concept de radargrammétrie (l'équivalent de la stéréovision avec le calcul de disparité en imagerie RSO), qui dans certains cas peut concurrencer l'interférométrie pour la reconstruction de MNT. Par rapport à ERS, la résolution est légèrement améliorée grâce à l'évolution des convertisseurs analogique-numérique. L'amélioration du temps de cycle a joué un rôle essentiel pour les territoires situés à latitude élevée sur lesquels une acquisition journalière est rendue possible grâce à l'agilité en incidence du capteur. Cependant, cette même agilité ne permet pas de constituer un catalogue d'acquisition systématique selon une modalité identique pour tous les points du globe.

\subsubsection{L'ESA et Le capteur ASAR d'ENVISAT (2002)}

En associant à l'approche $\mathbb{R S}$ le principe de l'an-tenne à focalisation électronique de Radarsat-1, ㅌESA voulait doter les scientifiques dun successeur aux $\mathbb{R S}$ permettant une incidence locale variable, un temps ce revisite plus bref, et quelques capacités polarimétriques. ENVISAT a donc été placé exactement sur la même orbite que les ERS, ce qui se traduit par le même cycle (35 jours). Malheureusement, la fréquence centrale étant très légèrement différente, il n'est pas possible de générer des interférogrammes entre ces deux capteurs : la continuité espérée entre les ERS et ENVISAT n'a pas été possible. Notons que ce manque de continuité des données a été sévèrement critiqué à l'époque et a été un des éléments clés du cahier des charges des futurs Sentinels.

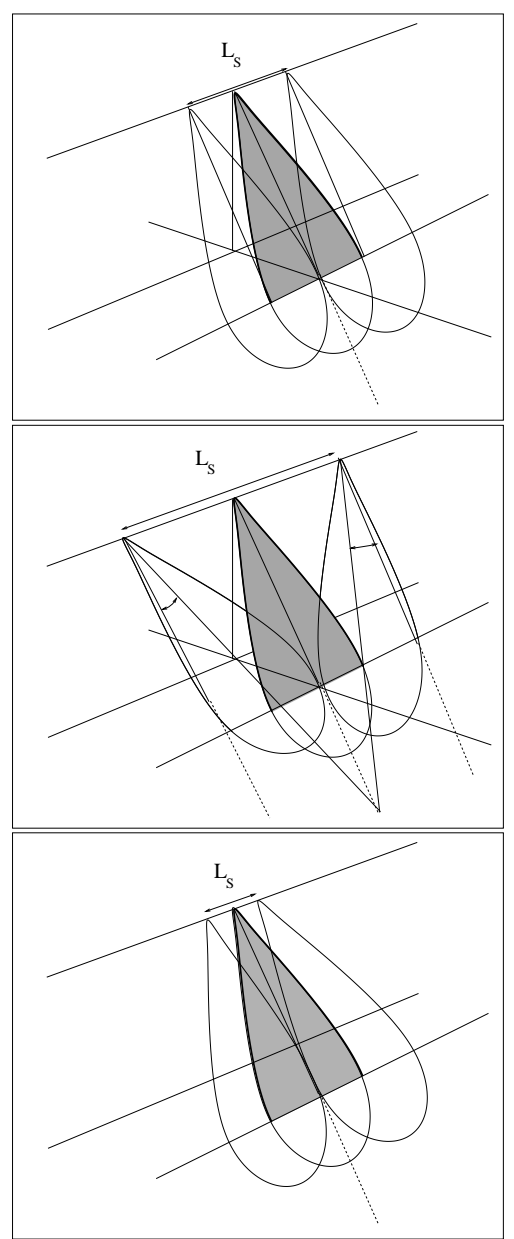

Figure 4 : Les capteurs æconde génération ont la faculté ce pouvoir cépointer leurs émissions ron sule-ment en modifiant l'angle d'émission (ce qui permet de modifier la localisation de la fauchée selon la distance), mais aussi en modifiant l'angle de squint. Cette modification peut se faire pour améliorer la résolution (au centre, mode Spotlight, pour lequel l'antenne synthétique LS est plus grande que le cas Stripmap) ou réduire le temps d'acquisition au détriment de la résolution (en bas, mode TOPSAR, pour lequel l'antenne synthétique LS est plus petite que le cas Stripmap). La figure du haut correspond au cas canonique du mode Stripmap ( $L S$ est donnée par la relation 3).

\subsection{La seconde génération de capteurs : 2006-2012}

Il a fallu attendre une quinzaine d'années après la mise en orbite d'ERS pour voir apparaître une nouvelle 
génération de capteurs RSO dont les performances ont été largement améliorées par rapport à la génération précédente, bénéficiant amplement de l'évolution des composants électroniques sur une grande décennie.

Les avancées scientifiques et technologiques ont provoqué un grand bond dans la résolution, qui s'améliore d'un facteur de l'ordre de la dizaine, ainsi que dans les nombreuses modalités d'acquisition possibles (comme les modes polarimétriques qui permettent d'accéder à une caractérisation fine des milieux rétrodiffusants).

Sont ainsi apparus en moins de quatre ans neuf satellites:

- ALOS (2006-2011) et ALOS-2 (2014), de l'agence spatiale japonaise, en bande $L$, qui ont été les premiers à proposer des données en pleine polarimétrie (à chaque pixel correspondent les quatre canaux $\mathrm{HH}$, HV, VH et VV);

- Radarsat-2 en 2007, de l'agence spatiale canadienne, en bande $C$ et en pleine polarimétrie ;

- TerraSAR-X (2007) et Tandem-X (2010) (auxquels il faut rajouter PAZ lancé en 2018), conçus par l'agence spatiale allemande (DLR) et exploités par Airbus.

Capteurs en bande $X$, leurs résolutions submétriques dans une modalité spécifique (SpotLight) ont permis aux images radar de pouvoir être à nouveau comparées avec les capteurs optiques (Ikonos, 1999, est à $1 \mathrm{~m}$ de résolution). La figure 6 montre la ville de Hoorn imagée avec TerraSAR-X (mode stripmap) : le pixel a une dimension de $2 \mathrm{~m} \times 2 \mathrm{~m}$.

- les quatre Cosmo-Skymed (de l'agence spatiale italienne, lancés entre 2007 et 2010), en bande X, qui permettent d'avoir au moins une image tous les huit jours (voire tous les quatre jours dans certaines configurations).

Si les progrès technologiques accumulés sur la décennie ont permis d'accéder à la résolution métrique et ainsi de s'approcher des résolutions des capteurs optiques, c'est peut être l'amélioration de la résolution temporelle qui est le fait le plus marquant. En effet, en prenant comme exemple le capteur TerraSAR-X, son cycle de 11 jours permet de construire rapidement des piles temporelles en configuration interférométrique : sur ces piles, d'une part il est possible de lisser le chatoiement présent par moyennage temporel et d'autre part d'analyser les variations de la cohérence interférométrique ce qui apporte des réponses nouvelles dans des problématiques très actuelles comme par exemple la détection de changement. Pour TerraSAR-X, on peut disposer de trois images par mois en configuration interférométrique sur un site donné. La philosophie des Cosmo-Skymed répond encore mieux à cette attente : en choisissant un cycle assez court (16 jours) et en mettant sur la même orbite une constellation de quatre capteurs identiques, il est possible d'avoir globalement 8 images par mois en configuration interférométrique (rappelons que pour un unique capteur comme ENVISAT, il n'y avait qu'une image de ce type tous les 35 jours). De surcroît, si l'on relâche la contrainte d'avoir des données en configuration interférométrique, la constellation des Cosmo-Skymed permet quasiment d'avoir au moins une image par jour de n'importe quel point de la planète.

Le second point marquant est l'agilité de l'antenne qui non seulement dépointe selon l'axe distance pour permettre de varier l'angle d'incidence mais aussi dépointe pour modifier la dimension de l'antenne synthétique. Cette modification peut s'appliquer aussi bien pour augmenter ou diminuer cette dimension :

- En recherchant une grande dimension d'antenne synthétique, on a la possibilité d'avoir des images de zones réduites mais avec une excellente résolution selon l'azimut : c'est le principe de l'acquisition Spotlight qui fournit des résolutions sub-métriques (par exemple $20 \mathrm{~cm}$ pour les données TerraSAR-X Staring Spotlight).

- En réduisant la taille de l'antenne synthétique, on peut acquérir une zone de la Terre en moins de temps, ce qui permet de balayer, sur un seul passage, plusieurs zones contigües permettant d'élargir la fauchée : c'est le mode TOPSAR (Terrain Observation with Progressive Scanning SAR). La figure 4 illustre ces deux différents modes d'acquisition.

Notons cependant que, comme dans le cas de l'imagerie optique, le gain en résolution se traduit par une diminution de la fauchée : par exemple une donnée CosmoSkymed Enhanced Spotlight (pixel de $70 \mathrm{~cm}$ ) a une fauchée de l'ordre de $10 \mathrm{~km}$ (une acquisition Pleiades couvre environ $20 \mathrm{~km}$ ). En revanche, dégrader la résolution conduit à des fauchées de grandes dimensions : le mode Wide ScanSAR de TerraSAR-X a une fauchée de $270 \mathrm{~km}$ pour une résolution devenue décamétrique.

II faut noter que l'Inde avec Risat-1 (2012) et Taïwan avec Kompsat-5 (2013), se sont aussi dotées de capteurs radar, montrant l'intérêt que peut apporter un capteur "tout temps" dans les zones du globe à forte couverture nuageuse.

\subsection{La révolution Sentinel (2014)}

Si la seconde génération de capteurs a vu une évolution marquée vers des résolutions meilleures, tant spatiale que temporelle, l'agilité des capteurs ne permet quasiment jamais de garantir des acquisitions systématiques selon des modalités d'acquisition identiques telles que pouvaient assurer des capteurs comme les ERS.

En choisissant de mettre en orbite deux satellites radar identiques (Sentinel-1A en 2014 et Sentinel-1B en 2016), de résolution intermédiaire (décamétrique), de durée de cycle réduit (12 jours) et de caractéristiques d'acquisition invariables pour un point donné du globe, l'ESA offre à la communauté scientifique des données tous les 6 jours, sous forme d'un catalogue de données multitemporelles à priori sans discontinuité temporelle. Ces données sont gratuites et accessibles par tous.

Pour arriver à une couverture globale dans un cycle aussi court, le mode TOPSAR a été choisi, permettant ainsi d'acquérir trois bandes lors d'un seul passage : la fauchée globale résultante est alors de l'ordre de 250 $\mathrm{km}$. Avec ce principe d'acquisition, on peut au fil des ans 
construire des piles temporelles sur lesquelles des traitements selon l'axe temporel peuvent être appliqués, en particulier des moyennages temporels qui filtrent le chatoiement sans changer la résolution. La figure 5 montre, toujours sur la ville de Hoorn, une image Sentinel-1 résultant du moyennage temporel d'une pile de 25 images TOPSAR/SLC acquises sur une année (voir le principe au paragraphe 2.3).

\section{Un demi siècle de traitements spécifiques à l'imagerie radar}

\subsection{L'étude statistique du chatoiement et les filtres dédiés à sa suppression}

\subsubsection{Le chatoiement, sa modélisation et son filtrage}

Le premier écueil à l'utilisation de données radar est la présence du chatoiement sur des régions à priori homogènes, et qui opère comme du bruit multiplicatif. $\mathrm{Ce}$ chatoiement peut se voir comme la résultante de l'interaction physique de l'onde avec la surface à l'échelle de la longueur d'onde.

Dans le cadre de l'imagerie cohérente (laser, acoustique, radar), Goodman (Goodman, 2007) a montré que la loi vérifiée par ce bruit est une loi de Rayleigh pour les données dites en amplitude. En privilégiant les données en intensité, la communauté scientifique a pu ainsi considérer que ce bruit suivait une loi exponentielle décroissante (cas particulier de la loi Gamma), celle-ci vérifiant la propriété d'additivité : la moyenne arithmétique calculée sur un voisinage spatial de $L$ valeurs (suppposées indépendantes) suit alors une loi Gamma paramétrée par le facteur $L$. En utilisant des valeurs ainsi moyennées (appelées aussi multivues), l'aspect de l'image devient moins granuleux : les données Seasat $(L=4)$ et ERS (données "PRl" avec $L=3$ ) ont utilisé cette propriété pour permettre une utilisation plus aisée par des non spécialistes. Des données suivent alors une loi Gamma (cas des données en intensité) ou une loi de Nakagami (cas des données en amplitude). Actuellement, les produits Sentinel géoréférencés ont un paramètre de multivue de l'ordre de 5 .

Les zones imagées peuvent en première étape être caractérisées par une texture. Cette texture peut se voir comme étant la variabilité de la rétrodiffusion de la surface à l'échelle de la résolution. En supposant un modèle de texture, les effets du chatoiement, qui agit comme un bruit multiplicatif, vont modifier le type de loi statistique de l'image : un certain nombre de travaux ont été dédiés à cet aspect des images et ont conduit à l'utilisation de lois plus complexes telles que la loi K, la loi de Fisher et la loi Gamma Généralisée. Un modèle global fondé sur la transformée de Mellin (Nicolas, 2002) permet d'unifier les approches et les méthodes d'estimation des paramètres. II permet aussi de définir des grandeurs utiles pour caractériser les lois, comme les log cumulants. On montre que ces lois ont leur log-cumulant d'ordre $2\left(\tilde{\kappa}_{2}\right)$ positif, et que le caractère de "loi à queue lourde" est lié à un log-cumulant d'ordre $3\left(\tilde{\kappa}_{3}\right)$ positif. Notons que la loi de Fisher est un exemple de "loi à queue lourde".
Connaissant la loi sous jacente pour la texture et le chatoiement, il est alors possible de proposer des filtres dédiés à la suppression du chatoiement : sont ainsi apparus les filtres de Kuan, de Lee, de Frost et bien d'autres, qui sont maintenant bien ancrés dans la panoplie des traitements applicables aux images radar (Tupin et al., 2013). Les données interférométriques et polarimétriques sont abordées selon la même philosophie : seule change la dimensionnalité du problème (qui peut varier selon que l'on traite des données Dual Pol -deux polarisations- ou Quad Pol -les 4 canaux de polarisation-).

\subsubsection{Quantification, texture et résolution : 40 ans d'évo- lution technologique et scientifique}

A ce stade, il est important de voir comment les évolutions technologiques des capteurs, tant sur le plan de la résolution que celui de l'électronique embarquée, ont eu des conséquences sur le traitement.

En effet, la caractéristique essentielle des images radar actuelles est d'avoir une grande dynamique : à côté de régions modélisables par une texture et sur lesquelles agit le chatoiement, d'autres régions vont se caractériser par des structures géométriques qui vont alors jouer un rôle majeur sur la rétrodiffusion. C'est par exemple le cas des immeubles orientés selon la trajectoire du satellite pour lesquels l'onde émise va effectuer un double rebond, le premier sur le mur de l'immeuble et le second sur le sol : l'énergie reçue par l'antenne est alors beaucoup plus grande que celle correspondant à un simple phénomène de chatoiement. Si l'on étudie le rapport entre l'énergie rétrodiffusée dans le cas du chatoiement et celle relative à un immeuble, il peut largement dépasser les $50 \mathrm{~dB}$. Ce phénomène s'observe aussi en présence de cibles ressemblant à des cibles dites canoniques (comme les coins cubes, appelés aussi corner reflector). Aussi la quantification des données est de la plus grande importance et est conditionnée par les performances du convertisseur analogique/numérique embarqué : les progrès des dernières décennies auront été essentiels aussi bien pour la qualité des données que pour celle des traitements. Remarquons toutefois que les plus célèbres RSO ont été les Venera 15 et 16 , en orbite autour de Venus (1983) : l'échantillonnage du signal reçu était effectué sur seulement 1 bit et a néanmoins permis la synthèse des premières images de la surface de cette planète recouverte de nuages.

A l'issue du traitement de synthèse, les données (en complexe ou en amplitude) sont généralement fournies codées sur 16 bits, voire en demi-float (données Tandem) : cette spécificité est indispensable. En effet, malgré les faibles performances des architectures informatiques de l'époque, les spécialistes des images ERS ont rapidement montré combien il était essentiel de garder la meilleure dynamique possible sur ce type de données : une analyse statistique, tant globale que locale, ne peut correctement aboutir si certains pixels brillants ont une valeur saturée parce que la dynamique de l'image, initialement sur 16 bits, a été ramenée sur 8 bits (c'est à dire la dynamique des images optiques de l'époque). En 


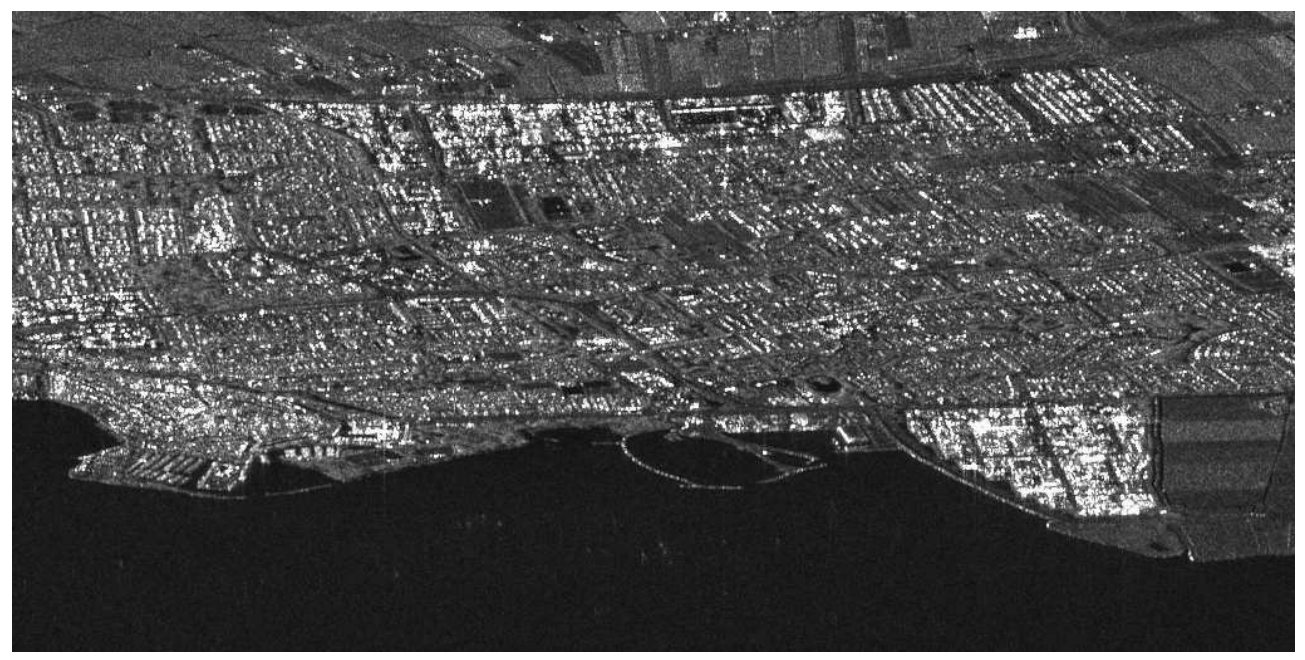

Figure 5 : Image de la ville de Hoorn obtenue à partir de données SLC/TOPSAR du capteur Sentinel-1 après moyennage multitemporel : le pixel est de $3.4 \mathrm{~m} \times 13.9 \mathrm{~m}$. L'imagette a pour dimension 1024×512 pixels.

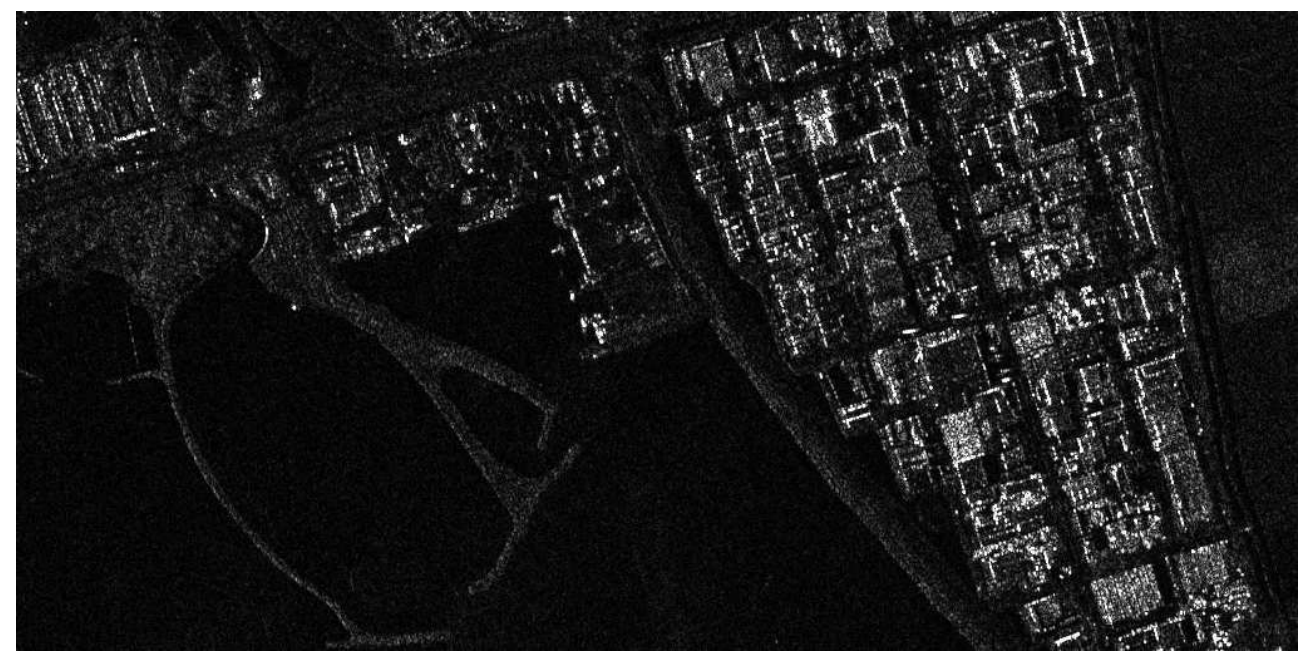

Figure 6 : Image de la ville de Hoorn obtenue par le capteur TerraSAR-X en mode strpmap (copyright DLR): le pixel est de $2 m \times 2 m$. L'imagette a pour dimension $1024 \times 512$ pixels.

ayant ainsi une plus grande dynamique, les modèles statistiques diffèrent alors une fois de plus de ceux de l'imagerie optique pour laquelle une dynamique de 8 bits a été longtemps suffisante.

Grâce à ces modèles statistiques plus évolués, les améliorations liées à la résolution peuvent être mises en évidence. Historiquement, le premier modèle de texture à être utilisé sur des données est celui de la loi Gamma (si on prend l'intensité de l'image) : l'image suit alors globalement une loi K (Jakeman et Pusey, 1976) et dans les années 70, cette approche a été largement utilisée et validée sur des systèmes terrestres ou aéroportés d'autant que les données étaient quasiment toujours ramenées sur 8 bits. Ce succès repose sur une propriété essentielle de la loi $\mathrm{K}$ : elle possède tous ses moments (ce type de loi peut être désigné comme "loi à tête lourde"). En aucun cas, la loi $\mathrm{K}$ ne peut être utilisée pour traiter des données ayant des données extrêmes (outliers) car celles-ci demandent à être modélisées par des lois dites "à queue lourde" (heavy tailed distributions). Néanmoins son application à des données de première génération (comme les images ERS) a été très judicieux.

En améliorant à la fois la résolution et la dynamique, les images radar ont requis des modèles de texture permettant des approches de type "loi à queue lourde", comme la loi de Fisher. En effet, au fur et à mesure que la cellule de résolution diminue en superficie (amélioration de la résolution), les effets liés à la présence éventuelle d'une cible canonique s'intensifient et les pixels prennent des valeurs les faisant de plus en plus passer pour des données extrêmes. La figure 7 illustre ce mécanisme en comparant l'allure de données ERS-SLC et de données TerraSAR-X SpotLight dans le diagramme $\tilde{\kappa}_{2}-$ $\tilde{\kappa}_{3}$ : les valeurs extrêmes correspondent à des valeurs du log-cumulant d'ordre $3\left(\tilde{\kappa}_{3}\right)$ positives. On en conclut que l'amélioration de la résolution se traduit par une forte augmentation du nombre de valeurs extrêmes, et qu'il est requis une forte dynamique pour les mettre correctement en évidence. Tout ceci montre l'importance du choix du modèle de texture sous jacente et du choix du modèle de 


\begin{tabular}{|c|c|c|c|c|c|c|c|c|c|}
\hline Satellite & Début-Fin & $\begin{array}{l}\text { altitude } \\
(\mathrm{km})\end{array}$ & $\begin{array}{c}\text { cycle } \\
\text { (jours) }\end{array}$ & $\begin{array}{l}\text { bande } \\
\text { radar }\end{array}$ & $\begin{array}{c}\lambda_{0} \\
(\mathrm{~cm})\end{array}$ & $\begin{array}{c}f_{0} \\
(\mathrm{GHz})\end{array}$ & & Antenne & $\begin{array}{c}B W_{\max } \\
(\mathrm{MHz})\end{array}$ \\
\hline \multicolumn{10}{|c|}{ RSO première génération } \\
\hline Seasat & 1978-1978 & 885 & 1717 & 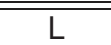 & 23.5 & 1.275 & $\mathrm{HH}$ & $10.7 \times 2.7$ & 19 \\
\hline ERS-1 & $1991-2000$ & 780 & 35 & $\mathrm{C}$ & 5.66 & 5.300 & $\mathrm{VV}$ & $10 \times 1$ & 15.55 \\
\hline ERS-2 & $1995-2011$ & 780 & 35 & C & 5.66 & 5.300 & VV & $10 \times 1$ & 15.55 \\
\hline ENVISAT & 2002-2012 & 780 & 35 & C & 5.62 & 5.331 & VV & $10 \times 1.3$ & 16 \\
\hline JERS & 1992-1998 & 568 & 44 & $\mathrm{~L}$ & 23.5 & 1.275 & $\mathrm{HH}$ & $12 . \times 2.2$ & 15 \\
\hline Radarsat-1 & $1995-2013$ & 798 & 24 & $\mathrm{C}$ & 5.66 & 5.300 & $\mathrm{HH}$ & $15 \times 1.5$ & 30 \\
\hline \multicolumn{10}{|c|}{ "RSO seconde génération } \\
\hline ALOS & $2006-2011$ & 692 & 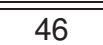 & 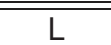 & 23.6 & 1.270 & FP & $8.9 \times 3.1$ & 28 \\
\hline ALOS-2 & 2014- & 628 & 14 & $\mathrm{~L}$ & 22.9 & 1.257 & FP & $9.9 \times 2.2$ & 84 \\
\hline TerraSAR-X & $2007-$ & 514 & 11 & $\bar{X}$ & 3.11 & 9.650 & $\mathrm{DP}$ & $4.8 \times 0.70$ & 300 \\
\hline Tandem-X & $2010-$ & 514 & 11 & $\mathrm{x}$ & 3.11 & 9.650 & DP & $4.8 \times 0.70$ & 300 \\
\hline CSK-1 à CSK-4 & $2007-$ & 619 & 16 & $\mathrm{X}$ & 3.12 & 9.600 & $\mathrm{M}$ & $5.7 \times 1.4$ & 400 \\
\hline Radarsat-2 & 2007 & 798 & 24 & $\mathrm{C}$ & 5.55 & 5.405 & FP & $15 \times 1.5$ & 100 \\
\hline RISAT-1 & 2012 & 536 & 25 & $C$ & 5.61 & 5.350 & $\mathrm{HP}$ & $6 \times 2$ & 225 \\
\hline Sentinel-1 & 2014 & 693 & 12 & $C$ & 5.55 & 5.405 & $\mathrm{DP}$ & $12.3 \times 0.82$ & 100 \\
\hline Kompsat-5 & 2013 & 550 & 28 & $\bar{X}$ & 3.11 & 9.66 & $\mathrm{M}$ & & 240 \\
\hline
\end{tabular}

Table 1 : Capteurs RSO les plus utilisés en imagerie satellitaire radar. L'indication FP dans la colonne de la polarisation correspond à Full Polar, DP à Dual Polar, et M signifie que l'acquisition de la monoimage peut se faire à une polarisation souhaitée (VV, VH, HV ou VV). RISAT-1 permet aussi une acquisition en polarisation circulaire (appelée aussi hybride, notée ici HP). Enfin notons que, hormis Seasat, ces satellites sont héliosynchrones.

loi globale : la dernière décennie a d'ailleurs montré l'utilité de lois spécifiques comme la loi Gamma Généralisée ou la loi de Fisher pour les données issues des capteurs $\mathrm{RSO}$ de seconde et de troisième génération.

\subsection{Nouvelles approches : filtres NL-SAR, SAR-SIFT}

De même que l'amélioration de l'électronique a permis des avancées majeures sur la résolution et l'agilité des capteurs radar, des méthodes récentes de traitement d'images ont leur rôle à jouer en imagerie radar. En effet celle-ci, bien qu'ayant des spécificités à prendre en compte, peut tirer parti des avancées significatives réalisées ces dernières années en traitement d'images et en vision par ordinateur. Nous allons nous concentrer sur deux exemples emblématiques que sont les approches par "patchs" (petits blocs de l'image) introduites par Buades et al. (Buades et al., 2005), et les SIFT, points caractéristiques de l'image auxquels sont associés des descripteurs proposés par Lowe et al. (Lowe, 2004). Notons que les travaux cités ici ne sont que des exemples parmi de nombreux autres (voir par exemple (Tupin et al., 2013).

\subsubsection{L'estimation des paramètres physiques et les ap- proches par patchs}

L'estimation des paramètres physiques (comme la réflectivité, la cohérence, ou encore la phase interférométrique) à partir des images radar est un problème ancien, rendu difficile par les statistiques du chatoiement suivies par ces données. Même si les distributions de ces paramètres sont relativement bien modélisées, il est néces- saire de disposer d'un grand nombre d'échantillons observés pour estimer les paramètres physiques de façon fiable. Ces échantillons sont généralement choisis localement, dans une fenêtre autour du pixel qu'on cherche à estimer. L'utilisation de grandes fenêtres va malheureusement à l'encontre de la résolution géométrique qu'on souhaiterait préserver.

Le principe des méthodes par patchs pour le débruitage consiste à sélectionner les échantillons à combiner pour faire l'estimation grâce à leur voisinage local. L'hypothèse fondamentale est que si leurs signaux locaux (les patchs) se ressemblent, alors les pixels véhiculent une information similaire. La sélection d'échantillons qui en résulte, qui s'appuie sur une distance entre patchs, est particulièrement robuste aux données bruitées, aspect déterminant pour le traitement des données radar.

Ces méthodes fonctionnent en 3 étapes principales : comparaison des patchs, combinaison des patchs pour l'estimation, affectation du résultat de l'estimation à chaque pixel. Celles-ci doivent bien sûr tenir compte des statistiques des données RSO. Tout d'abord, la comparaison de patchs doit être adaptée. La distance euclidienne qui est efficace dans le cas d'un bruit additif gaussien doit être remplacée par exemple en s'appuyant sur un test d'hypothèses. La combinaison des patchs doit également tenir compte des distributions, une solution étant d'utiliser un maximum de vraisemblance pondéré des observations. Finalement, différentes stratégies de recombinaison sont possibles.

Une discussion détaillée sur celles-ci peut être trouvée dans (Deledalle et al., 2014a). L'intérêt de ce cadre 

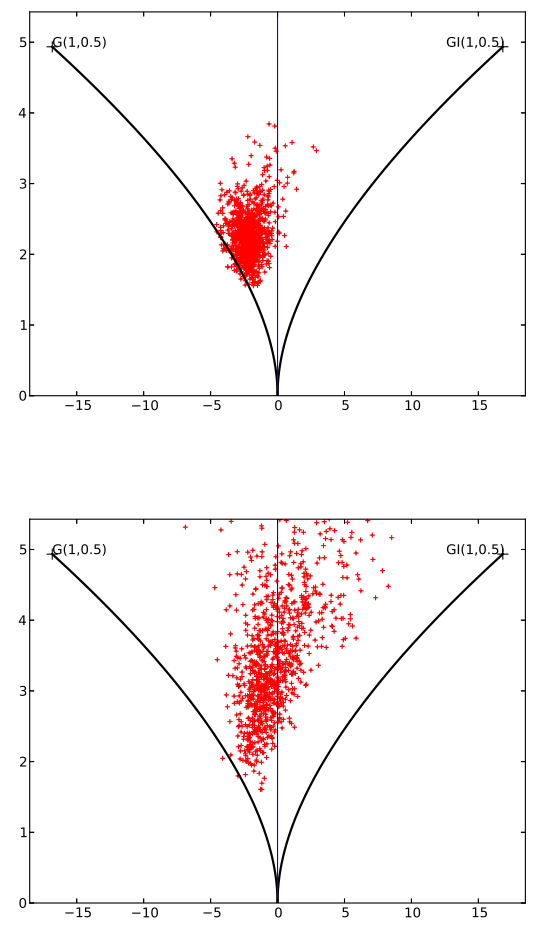

Figure 7 : Analyse comparative du diagramme $\tilde{R} 2-\tilde{K} 3$ entre une image ERS (en haut) et une image TerraSAR-X Spotlight (en bas) acquises sur la même zone de Paris Intra muros. En présence de seul chatoiement, les nuages seraient concentrés autour du point théo-rique correspondant à une loi de Rayleigh placée sur la branche de gauche (le lieu des lois de Nakagami). La branche de droite correspond aux lois de Nakagami In-verse, qui sont des lois à queue lourde par excellence.

général est qu'il peut s'appliquer pour l'estimation ds différents paramètres physiques d'intérêt que ce soit la réflectivité al la phase interférométrique.

\subsubsection{Les points d'intérêt et les descripteurs pour l'ima- gerie RSO}

De la même façon que les approches par patchs ot révolutionné les approches de débruitage, les \$FT proposés par lowe $\&$ al. (Lowe, 2004) ot el un impact déterminant en vision par odinateur pour la mise en correspondance entre images. @mme pour les approches précédentes, l'élément dé est la modélisation dun voisinage local, capturant les informations structurelles à échelle fine. Afin dintroduire des propriétés dinvariance en échelle \& en orientation, le voisinage est analysé par rapport à une oientation locale principale \& selon une taille dépendant de l'échelle locale caractéristique. De plus, pour introduire ces propriétés dinvariance radio-métrique, le descripteur est céfini par l'intermédiaire ce la dstribution ces gradients locaux. Les points dés \& les descripteurs associés calculés par cette approche sont très stables \& permettent des mises en correspondance dans des situations complexes comprenant des céforma-tions radiométriques \& géométriques.

De la même façon que précédemment l'utilisation de ces approches nécessite quelques adaptations compte tenu des spécificités du bruit. A nouveau, le cadre statistique et la bonne modélisation du chatoiement permettent d'adapter les différentes étapes, notamment en introduisant des gradients s'appuyant sur le logarithme pour obtenir des performances indépendantes de la radiométrie moyenne (voir par exemple les SAR-SIFT proposés dans (Dellinger et al., 2015)).

Ces approches permettent des mises en correspondance robustes, par exemple entre capteurs RSO de types différents. Un exemple d'application de SAR-SIFT est montré sur la figure 9.

\subsection{Le recalage des données RSO et les filtres sur piles temporelles}

Pour utiliser des données multi-capteur (même date, comme dans le cas de TerraSAR-X et Tandem-X), ou multi-passe (même capteur à des dates différentes), voire même multi-capteur multi-passe, une étape essentielle est de recaler les données dans le même référentiel (généralement celui de l'une des données). Deux grandes philosophies se dégagent à l'aune de l'histoire des satellites radar:

- Pour les capteurs de première génération, la précision à priori sur les orbites était insuffisante pour permettre ce recalage sans traitement spécifique. Les étapes de recalage devaient alors s'appuyer sur des techniques avancées de traitement d'images, voire sur l'utilisation de points d'amer.

- Pour les capteurs les plus récents, la position du capteur est extrêmement bien connue, ce qui permet directement de recaler deux images par simple connaissance des paramètres d'acquisition et des descripteurs d'orbite. Ce résultat assez extraordinaire a été en pratique acquis grâce aux nombreuses expérimentations d'orbitographie menées sur les capteurs de première génération, comme l'instrument DORIS

(Doppler Orbitography and Radio-positioning Integrated by Satellite), embarqué entre autres sur les ERS et ENVISAT. II faut noter qu'une fois de plus la qualité des horloges embarquées joue un rôle essentiel dans cette méthode.

II n'en reste pas moins que le rééchantillonnage des données à valeurs complexes est une étape délicate mais indispensable pour que, sous l'hypothèse d'un sol plat, les pixels correspondent à la même zone du sol. En particulier, pour les modes Spotlight et Topsar, des traitements spécifiques (l'étape de deramping en particulier) sont requis pour prendre en compte la variation de squint durant l'acquisition. Cependant, le fait de traiter les données SLC permettent un rééchantillonnage de bien meilleure qualité que si l'on traitait les mêmes données en amplitude : ceci justifie la préférence que l'on doit apporter aux données complexes vis à vis de données en amplitude (ceci explique le choix des données Sentinel1 SLC dans l'exemple de la figure 5).

Une fois les données bien recalées (si possible avec une précision proche du dixième de pixel), plusieurs approches sont alors exploitables: 

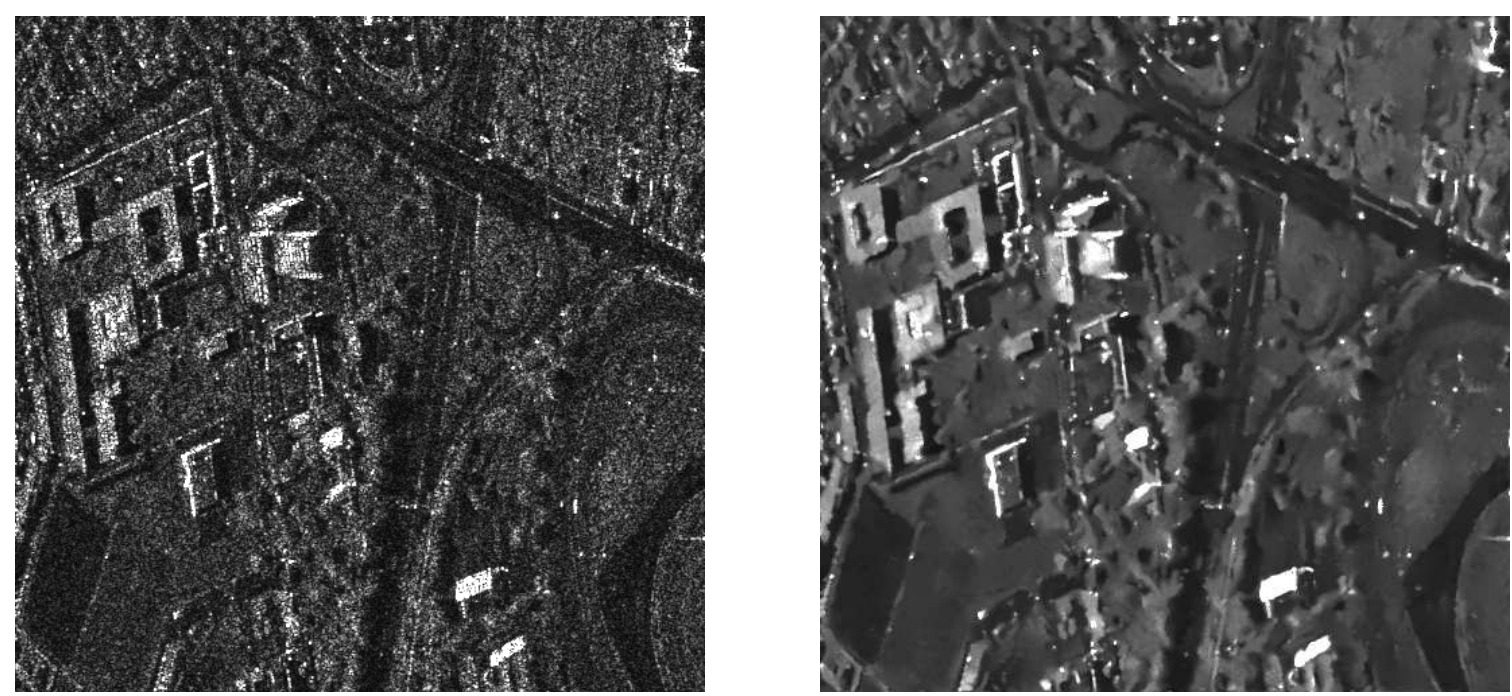

Figure 8 : Image TerraSAR-X de Toulouse (Copyright DLR) A gauche : image radar originale, mode Spotlight, résolution métrique ; à droite, résultat de l'estimation de l'amplitude par auto-similarité dans l'image obtenu par NL-SAR (Deledalle et al., 2014b).
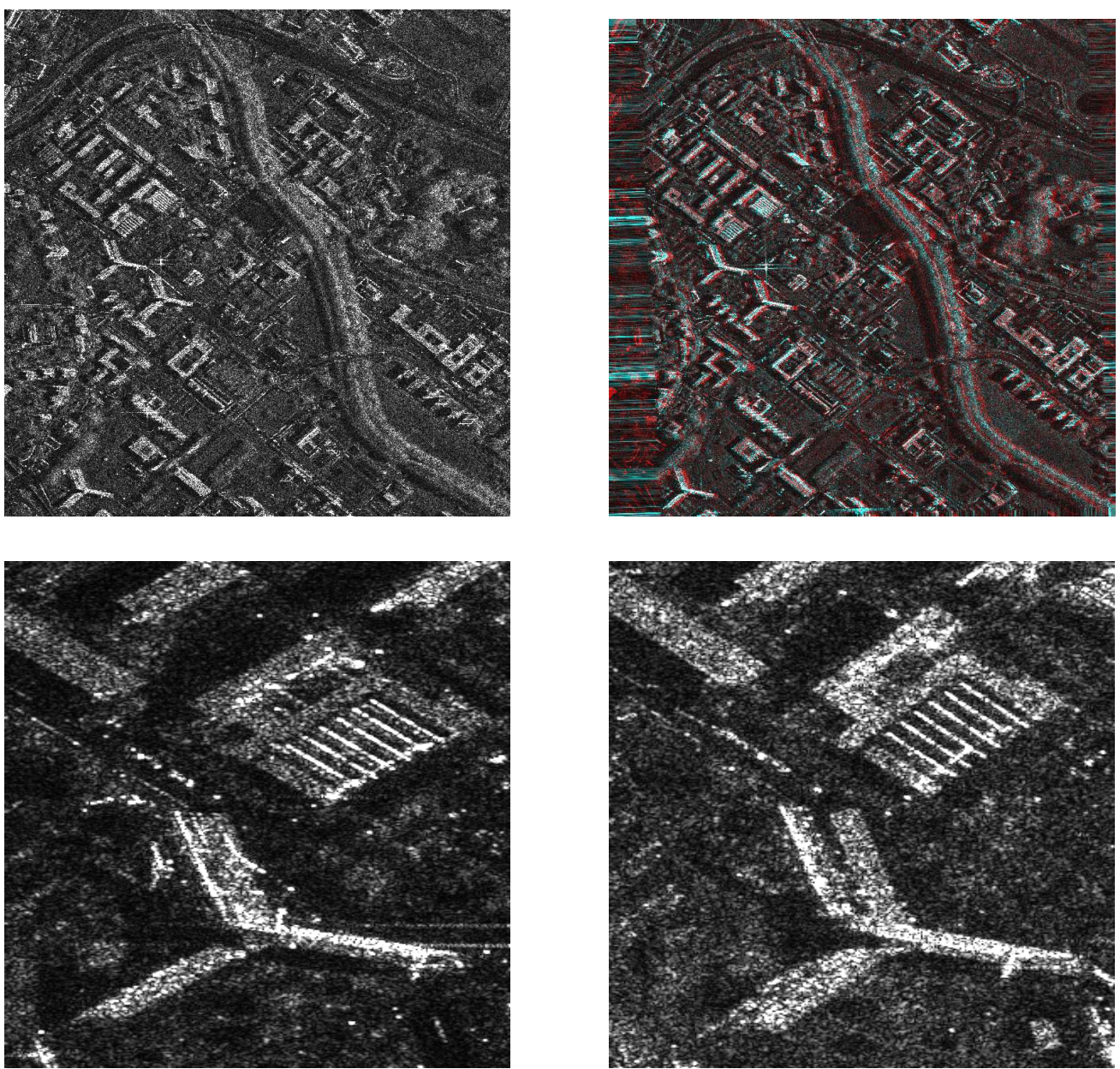

Figure 9 : Exemple de recalage automatique avec SAR-SIFT (Dellinger et al., 2015). En haut à gauche, image TerraSAR-X originale (angle d'incidence $48^{\circ}$ ); en haut à droite superposition des deux images recalées (les angles d'incidence sont respectivement de $48^{\circ}$ et $34^{\circ}$, cette différence apparaissant clairement dans les zooms des images en bas). Le recalage est effectué par recherche de la déformation appariant le plus grand nombre de SAR-SIFT, et correspond donc aux points situés au sol. Les variations de couleur montrent le décalage correspondant à l'élévation des bâtiments. 
En supposant que l'information est stable, on peut traiter les images par des outils de filtrage de chatoie-ment temporel (comme une simple moyenne géomé-trique) : la "super-image" ainsi obtenue devient une image sans chatoiement, ceci sans perte de résolu-tion (voir figure 5).

- En comparant les phases des deux images : c'est le principe de l'interférométrie, qui nécessite que les positions des deux capteurs soient assez proches et que les rétro-diffuseurs au sol demeurent à peu près inchangés. Puisque la géométrie d'acquisition est maintenant bien connue sur les satellites actuels, toute différence entre image, qui s'observe sous forme d'une différence de phase, est due à une méconnaissance du MNT, à une méconnaissance du sursol, ou à un mouvement de la croûte terrestre (mouve-ment rapide lié à un séisme ou mouvement lent de subsidence). A ce degré de finesse de déplacement, il est nécessaire d'appliquer des corrections sur la différence des couches atmosphériques entre deux acquisitions dans le cas de données multipasse.

- En comparant les variations d'amplitudes ou la qualité de l'interférogramme (données de cohérence), on détecte les changements. Cette comparaison ouvre le champ à de très nombreuses applications, devenues possibles grâce au flux important de données lié au faible temps de revisite actuel.

\section{Conclusion}

Près d'un demi-siècle s'est écoulé depuis le lancement et l'acquisition de données radar sur notre Terre. Trois générations de capteurs se sont succédées, améliorant la qualité des données selon des pistes diverses : résolution, temps de revisite, qualité du positionnement des satellites, accès gratuit aux données,... Les prochains satellites (Biomass, SWOT) illustrent aussi la vi-talité du domaine en proposant des capteurs originaux et dédiés à des applications phare du suivi de notre planète (hydrographie, végétation).

II est certain que le caractère "tout temps" des don-nées RSO les rend indispensables au suivi de notre pla-nète. Cependant leur lisibilité, fortement perturbée par le chatoiement omni-présent, est un obstacle à une dif-fusion plus massive et généralisée, les images optiques ayant toujours plus de sens pour un opérateur humain.

A l'heure des Sentinels (tant les deux satellites radar que les deux satellites optique), la communauté scientifique dispose de données acquises selon la même modalité avec un temps de revisite suffisament court pour générer de grandes piles de données dont la comparaison peut être quasi immédiate puisque les images sont superposables. Détection de changement, suivi des mouvements de la croûte terrestre, suivi des activités anthropiques, suivi de la végétation sont alors directement ac-cessibles et utilisables.

Cependant, ces domaines d'application et d'exploitation de données ne doivent pas faire oublier que certains sujets de recherche spécifiques à l'imagerie radar sont toujours d"actualité : fusion optique-radar, fusion radar multi-passes et multi-capteurs.
Pour des données ac-quises par des satellites agiles, la variabilité d'aspect selon la direction de prise de vue et l'angle d'incidence est un frein à toute comparaison simple et immédiate. C'est probablement un challenge à la portée des chercheurs actuels que de lever cet obstacle, rendant les données RSO accessibles à tous et en leur permettant ainsi une très large diffusion.

\section{Références}

Buades, A., Coll, B., Morel, J.-M., 2005. A review of image denoising algorithms, with a new one. SIAM journal on Multiscale Modeling and Simulation 4 (2), 490.

Deledalle, C.-A., Denis, L., Poggi, G., Tupin, F., Verdoliva, L., juillet 2014a. Exploiting patch similarity for SAR image processing. IEEE Signal Processing Magazine 31 (4), 69-78.

Deledalle, C.-A., Denis, L., Tupin, F., Reigber, A., Jäger, M., 2014b. NL-SAR : a unified non-local framework for resolutionpreserving (Pol)(In)SAR denoising. IEEE Transactions on Geoscience and Remote Sensing 53 (4), 2021 - 2038.

Dellinger, F., Delon, J., Gousseau, Y., Michel, J., Tupin, F., 2015. SAR-SIFT : a SIFT-like algorithm for SAR images. IEEE Transactions on Geoscience and Remote Sensing 53 (1), 453 466.

Goodman, J., 2007. Speckle phenomena in optics : Theory and applications. Roberts \& Company.

Jakeman, E., Pusey, N., 1976. A model for non Rayleigh sea echo. IEEE Trans. on AP 24 (6), 806-814.

Lowe, D. G., 2004. Distinctive image features from scaleinvariant keypoints. Int. J. Comput. Vision 60, 91-110.

Massonnet, D., Souyris, J., 2008. Imaging with Synthetic Aperture Radar. EPFL Press.

Nicolas, J.-M., 2002. Introduction aux statistiques de deuxième espèce : applications des logs-moments et des logscumulants à l'analyse des lois d'images radar. Traitement du signal 19 (3), 139-167.

Tupin, F., Nicolas, J.-M., Inglada, J., 2013. Imagerie de Télédétection. Hermes. 